\title{
LE GÉRONDIF COMME COMPLÉMENT DES VERBES DE PERCEPTION DANS LE PORTUGAIS BRÉSILIEN
}

\author{
Patrícia Araújo Rodrigues*
}

\section{Introduction $^{* *}$}

$\mathrm{N}$

ous nous proposons d'examiner dans cet article les compléments des verbes de perception construits avec un gérondif dans le portugais brésilien (PB), dont un exemple apparaît en (1):

(1)a) Maria viu as crianças brincando no jardim.

b) Maria a vu les enfants jouant dans le jardin.

Les constructions gérondives du PB équivalent aux constructions infinitives prépositionnelles très fréquentes dans le portugais européen (PE), dont un exemple est donné en (2) (Maurer, 1968; Gonçalves, 1999):

(2)a) Maria viu os meninos a brincar no jardim.

b) Maria a vu les garçons à jouer dans le jardin.

* Université du Quebec à Montreal.

** J'aimerais remercier l'Université du Québec à Montréal et le Conseil de recherches en sciences humaines du Canada, ainsi que le fonds FCAR, pour leur soutien financier. 
Raposo (1989) a étudié les propriétés syntaxiques des infinitives prépositionnelles compléments d'un verbe de perception. Selon lui, ces constructions ont la structure $\mathrm{NP}_{1} \mathrm{~V}\left[\mathrm{NP}_{2} \mathrm{a} \ldots \mathrm{VP}\right]$, où la séquence $\mathrm{NP}_{2} \mathrm{a} \ldots \mathrm{VP}_{\text {infin }}$ est un constituant unique sélectionné par le verbe de perception.

Guasti (1992 a,b) compare les infinitives prépositionnelles du PE à un autre type de complément des verbes de perception, à savoir, les pseudorelatives (un NP suivi d'une relative prédicative), ${ }^{1}$ dont un exemple apparaît en (3) :

(3)a) Vejo Maria que sai de casa.

b) (Je) vois Maria qui sort de chez elle.

Elle conclut que ces deux types de constructions partagent plusieurs caractéristiques sémantiques et syntaxiques, et peuvent, par conséquent, être analysées de la même façon. Pour Guasti, ces constructions sont formées par un constituant unique; elles constituent une petite proposition dominée par un nœud CP. Le complémenteur (que ou $a$ ) est la tête de la construction et le NP initial est dans la position de spécifieur de CP.

Proposition dominée par un noud CP. Le complémenteur (que ou $a$ ) est la tête de la construction et le NP initial est dans la position de spécifieur de CP.

Labelle (1996), contrairement à Guasti (1992a,b), analyse les pseudorelatives comme étant formées par deux constituants distincts, soit un objet suivi d'un prédicat second.

Les compléments gérondifs d'un verbe de perception sont fréquents dans des langues autres que le portugais, comme l'espagnol et l'anglais. Plusieurs analyses ont été proposées pour les compléments au gérondif en anglais (Akmajian, 1977; Cinque, 1991; Declerck, 1981, 1982; Felser, 1998; Guasti, 1992a, 1992b; Higginbotham, 1983; Safir, 1993). Selon Declerck (1982), suivi par Cinque (1991) et Felser (1998), ces différentes analyses résultent du fait que ces constructions sont ambiguës et correspondent en fait à trois constructions différentes, devant donc recevoir trois analyses différentes. Pour Declerck, ces constructions (voir l'exemple en (4)) correspondent soit à un constituant de type NP complexe (le NP initial est la tête de la construction et la participiale est un "pseudo-modifieur"), soit à une

1 Pour une discussion des différences structurales entre les pseudorelatives et les relatives, voir Guasti (1992a). 
RODRIGUES, P. A. Le gérondif comme complément des verbes...

séquence constituée d'un NP objet direct suivi d'un adjoint participial, ou soit à un constituant unique dénotant un événement (single clausal constituent). ${ }^{2}$

(4) I saw the moon rising over the mountain.

Borgonovo (1998) a comparé les constructions espagnoles où le complément d'un verbe de perception est constitué d'un NP et d'un gérondif aux constructions anglaises de même type:

(5)a) I saw Pedro dancing a mazurca.

b) Lo vi a Pedro bailando una mazurca.

Pour cet auteur, la construction anglaise (5a) correspond à deux structures différentes : i) le NP est le seul argument du verbe et le gérondif est un dépictif adjoint; ii) le NP et le gérondif forment un seul constituant qui reçoit l'unique rôle thématique interne du verbe. La construction espagnole (5b), par contre, n'est pas ambiguë, ne recevant qu'une structure, soit celle décrite en (i).

En français aussi, nous trouvons ce même type de complément des verbes de perception, c'est-à-dire un complément construit avec un NP et un gérondif. Toutefois ces constructions ne sont pas d'usage fréquent dans la langue, les locuteurs préférant l'utilisation des compléments infinitifs. Ce type de construction française n'est pas mentionnée dans la littérature que nous avons consultée concernant les verbes de perception.

Ces faits nous amènent à poser une première question, à savoir si les constructions gérondives du PB sont ambiguës, comme celles de l'anglais, ou non ambiguës, comme celles de l'espagnol. Autrement dit, nous voulons déterminer combien de structures correspondent à cette construction. De plus, nous aimerions savoir quelles seraient les interprétations qui peuvent leur être associées; plus spécifiquement, nous nous demandons si ces constructions peuvent être analysées d'une part de la même façon que les pseudorelatives, telles que les infinitives prépositionelles du PE, et d'autre part de la même façon que les infinitives. Pour ce

2 Dans l'analyse de Cinque (1991), ce constituant unique est un CP à la tête vide. 
RODRIGUES, P. A. Le gérondif comme complément des verbes...

faire, nous nous basons principalement sur les divers tests et instruments d'analyse utilisés par Labelle (1996) dans son étude sur la complémentation du verbe voir.

\section{Les propriétés des compléments au gérondif des verbes de perception}

Les verbes de perception désignent le mode sensoriel à travers lequel un individu perçoit l'événement codé dans leurs compléments et expriment la relation existante entre l'expérienceur et l'événement ou objet perçu. L'interprétation du verbe perceptif est dépendante du contexte de perception, qui peut être réel ou hypothétique. Dans le contexte réel, la perception est visuelle (dans le cas de voir) et nous avons une lecture du verbe de perception que nous appellerons " concrète". Dans le cas d'un contexte irrealis ou hypothétique, la perception se passe plutôt dans l'imagination de l'expérienceur et nous disons que le verbe perceptif a une lecture imaginative. On peut alors paraphraser l'énoncé en remplaçant le verbe voir par le verbe imaginer. Dans ce travail nous considérons les verbes de perception dans un contexte réel. ${ }^{3}$ Nous reviendrons sur la notion de perception imaginative et sur ses implications dans l'interprétation des constructions perceptives à la fin de cette section.

Le verbe voir peut sélectionner différents types de compléments, par exemple les compléments à temps fini, les infinitives, les pseudorelatives ou les compléments construits avec un gérondif. Dans les études comparant les compléments à temps fini aux compléments à l'infinitif, les auteurs sont généralement d'accord pour affirmer que ces deux types de compléments possèdent des caractéristiques distinctes et qu'ils doivent nécessairement correspondre à des structures différentes. Les compléments à temps fini constituent une proposition, donc un CP. Les compléments à l'infinitif correspondent à un événement, donc à un IP. ${ }^{4}$ Dans le cas des pseudorelatives, comme nous l'avons vu dans l'introduction, des analyses différentes ont été proposées : elles seraient formées soit d'un constituant unique, soit de deux constituants (un NP suivi d'un prédicat second).

3 Parmi nos exemples, il y en a qui ne sont pas agrammaticaux si l'on considère l'interprétation imaginative du verbe voir. Toutefois, nous les précéderons d'un '*' pour indiquer qu'ils sont agrammaticales sous une lecture concrète.

4 Pour Safir (1993), le complément à l'infinitif peut être un IP ou un VP, selon les propriétés de sélection du verbe de perception. 
Pour caractériser les propriétés sémantiques et syntaxiques des compléments au gérondif des verbes de perception, nous comparons dans les prochains paragraphes leurs caractéristiques à celles des compléments à temps fini, ainsi qu'à celles des compléments à l'infinitif et des pseudorelatives.

\section{Perception directe et indirecte}

Dans le cas du verbe voir, nous parlons de perception directe lorsque l'événement ou l'objet codé dans le complément de ce verbe est perçu directement, c'est-à-dire ce qui est perçu (et décrit) est nécessairement dans le champ de vision de l'expérienceur de la perception. Nous parlons de perception indirecte lorsque la perception est le résultat d'une activité inférentielle basée sur des indices perçus directement, c'est-à-dire ce qui est décrit n'est pas immédiatement visible, mais est plutôt inféré à partir de certains indices se rapportant à la situation décrite.

L'utilisation de l'expression pelo seu jeito 'à son air' nous permet de distinguer les deux types de perception. En effet, cette expression indique que le résultat de la perception est inféré à partir d'un indice, l'air triste ou fâché de Maria, par exemple. Elle indique donc que l'événement n'a pas été perçu directement. Ainsi, l'exemple en (6) nous montre que les constructions avec un complément à temps fini n'impliquent pas la perception directe, et suggèrent plutôt la perception indirecte : l'expression pelo seu jeito 'à son air' ne rend pas la phrase agrammaticale. Par contre, les constructions avec un complément à l'infinitif ne peuvent être utilisées que pour décrire ce qui est perçu directement : si la perception est basée sur des indices de l'événement plutôt que sur la perception directe de l'événement même, la phrase est agrammaticale, comme on peut le constater en (7). Selon Guasti (1992 a, 1992 b), ceci est aussi le cas des pseudorelatives et des infinitives prépositionnelles, qui impliquent toujours la perception directe. Les constructions avec un complément au gérondif (voir (8)) se comportent comme celles avec un complément à l'infinitif, une pseudorelative ou une infinitive prépositionnelle, c'est-à-dire qu'elles impliquent la perception directe.

(6) a) Eu vi (pelo seu jeito) que Maria brigou com seu filho.

b) J'ai vu (à son air) que Maria s'est disputée avec son fils.

(7)a) Eu vi (*pelo seu jeito) Maria brigar com seu filho.

b) J'ai vu (*à son air) Maria se disputer avec son fils. 
RODRIGUES, P. A. Le gérondif comme complément des verbes...

(8) a) Eu vi (*pelo seu jeito) Maria brigando com seu filho.

b) J'ai vu (*à son air) Maria se disputant avec son fils.

\section{Les restrictions sémantiques sur le prédicat complément}

Il a été souvent remarqué que, dans le cas de l'interprétation concrète du verbe de perception, les constructions comportant un complément à l'infinitif excluent les prédicats statifs ${ }^{5}(9 \mathrm{a}, \mathrm{b})$. Les constructions comportant un complément à temps fini, par contre, ne présentent pas de restrictions sémantiques sur le prédicat subordonné $(10 \mathrm{a}, \mathrm{b})$. Les pseudorelatives $(11 \mathrm{a}, \mathrm{b})$ et les compléments au gérondif (12 a,b) (toujours dans le cas de l'interprétation concrète de voir) se comportent comme les compléments à l'infinitif, présentant aussi des restrictions concernant les verbes statifs.

(9) a) *Eu vejo Maria possuir uma casa.

b) *Je vois Maria posséder une maison.

(10) a) Vejo que Maria possui uma casa.

b) Je vois que Maria possède une maison.

(11) a) *Eu vejo Maria que possui uma casa.

b) *Je vois Marie qui possede une maison.

(12) a) *Eu vejo Maria possuindo uma casa.

b) *Je vois Marie possédant une maison.

Cependant, on a aussi remarqué que certains verbes statifs peuvent apparaître dans les constructions à l'infinitif lorsqu'elles décrivent un état transitoire, comme les exemples en (13) (Felser, 1998).

5 Selon la classification de Vendler (1967), les prédicats statifs sont ceux qui indiquent une durée ou persistance à travers le temps et n'impliquent ni dynamisme ni changement. 
(13) a) ?We saw John own a car (for five minutes once).

b) We saw John be obnoxious (again).

Ce fait a amené plusieurs auteurs, comme Boivin (1998), Felser (1998) et Guasti (1992 a), à suggérer que la description des contraintes sémantiques sur les prédicats infinitifs compléments des verbes de perception doit se baser plutôt sur la distinction proposée par Carlson (1977) entre les prédicats d'individus (individuallevel) et les prédicats d'un stage de l'individu (stage-level). Pour ces auteurs, les prédicats dans les compléments à l'infinitif des verbes de perception doivent, pour que l'interprétation concrète soit disponible, se restreindre aux prédicats stage-level. C'est aussi le cas pour les compléments au gérondif des verbes de perception en PB : les prédicats individual-level ne permettent pas la lecture concrète du verbe de perception.

\section{Le temps}

Les compléments à temps fini ne manifestent pas de restrictions temporelles; le temps dans ces subordonnées peut être différent de celui de la principale, tel qu'illustré par l'exemple en (14). Dans les phrases comportant un complément à l'infinitif, cependant, le temps de la subordonnée est dépendant de celui de la principale, comme nous le montre l'exemple en (15).

(14) a) Maria vê que os meninos brincaram no jardim ontem.

b) Maria voit que les garçons ont joué dans le jardin hier.

(15) a) *Maria vê os meninos brincar no jardim ontem.

b) Maria voit les garçons jouer dans le jardin hier.

Dans le cas des pseudorelatives, Guasti (1992 a, b) observe qu'elles aussi présentent des restrictions temporelles, le temps dans la subordonnée devant être le même que celui de la principale: 
RODRIGUES, P. A. Le gérondif comme complément des verbes...

(16) a) *Vedo Maria che parlava con Gianni.

(Je) vois Maria qui parlait avec Gianni.

b) *Ho visto Maria che parla con Gianni.

(Je) ai vu Maria qui parle avec Gianni.

Les compléments gérondifs en PB présentent également des restrictions concernant le temps de la subordonnée; il est dépendant de celui de la principale:

(17) a) *Maria vê os meninos brincando no jardim ontem.

b) Maria voit les garçons jouant dans le jardin hier

\section{Présupposition}

Selon Labelle (1996), dans les constructions comportant un complément à temps fini, comme en (18 a), la situation perçue est présupposée, c'est-à-dire que sa vérité est supposée par le locuteur. Dans le cas des compléments à l'infinitif, comme en (18 b), la situation perçue n'est pas présupposée, sa vérité n'est pas nécessairement supposée par le locuteur. Ce type de complément n'a pas une valeur de vérité indépendante de celle de la principale.

a) Pierre a vu qu'une soucoupe volante avait atterri dans son jardin.

: présuppose une soucoupe volante a atterri dans son jardin.

b) Pierre a vu une soucoupe volante atterrir dans son jardin.

: ne présuppose pas une soucoupe volante a atterri dans son jardin.

Nous pouvons comparer les compléments gérondifs du PB aux compléments à l'infinitif en ce qui concerne la véridicité de la subordonnée. La valeur de vérité des compléments au gérondif n'est pas indépendante de celle de la principale, contrairement aux compléments à temps fini, qui ont une valeur de vérité indépendante de celle de la principale. Ainsi, lorsqu'on énonce la phrase apparaissant 
en (19 a), où le complément est construit avec un gérondif, on ne peut pas supposer la vérité de la situation perçue; ce n'est pas le cas pour la phrase en (19 b), où nous avons un complément à temps fini.

a) Pierre viu um disco voador pousando no seu jardim. Pierre a vu une soucoupe volante atterrissant dans son jardin.

b) Pierre viu que um disco voador pousou no seu jardim. Pierre a vu qu'une soucoupe volante avait atterri dans son jardin.

\section{Perception imaginative}

Comme nous l'avons mentionné au début de cette section, le verbe voir peut avoir une interprétation qu'on appelle imaginative, lorsque le contexte d'interprétation est irrealis ou hypothétique. Sous une lecture imaginative, le verbe voir n'impose pas les mêmes restrictions que nous avons discutées dans le cas de la lecture concrète en ce qui concerne le type aspectuel ou le temps du verbe de la subordonnée.

Le type aspectuel du verbe subordonné dans un contexte irrealis

En français, selon Boivin (1998), nous pouvons avoir la présence d'un prédicat individual-level dans le complément infinitif du verbe voir lorsqu'il a une interprétation imaginative. Autrement dit, le prédicat individual-level dans les compléments infinitifs force une lecture imaginative (non-visuelle), tel qu'illustré par l'exemple donné en (20) (Boivin, 1998, p.107); pour l'auteur, cette phrase est agrammaticale si on lui attribue une interprétation concrète, mais est grammaticale sous une lecture imaginative. 
(20) Je vois Jean connaître l'histoire du monde.

Selon Boivin, l'anglais se comporte différemment du français, en ce sens que les compléments infinitifs en anglais se restreignent aux prédicats stage-level. Ces compléments sont donc toujours associés à une interprétation concrète du verbe de perception, comme nous pouvons le constater en examinant les exemples en (21). Contrairement au français, une interprétation imaginative est impossible en (21a). Pour qu'une interprétation imaginative soit obtenue, le verbe subordonné doit avoir la forme du gérondif, comme en (22). Cette phrase peut aussi avoir une interprétation concrète : les compléments gérondifs créent donc une ambiguïté lorsqu'il s'agit de prédicats stage-level. Par ailleurs, ce type de complément admet, contrairement au complément à l'infinitif, un prédicat individual-level, qui force toutefois l'interprétation imaginative (voir (23)).

a) I see John play the guitar.

b) *I see John know the history of the world.

(22) I see John playing the guitar.

(23) I see John knowing the history of the world.

Boivin ne traite pas des constructions françaises où un verbe de perception sélectionne un complément au gérondif.

Quant au PB, il se comporte comme l'anglais. C'est-à-dire que le complément gérondif est ambigu par rapport aux interprétations concrète et imaginative dans le cas des prédicats stage-level (voir (24)). Dans le cas des prédicats individual-level, ce type de complément n'est pas ambigu et force une interprétation imaginative (voir (25)). Le complément à l'infinitif n'est pas compatible avec les prédicats individual-level (voir (26a)) et n'est pas ambigu avec les prédicats stage-level, forçant toujours une interprétation concrète (voir (26b)).

(24) Maria vê os meninos brincando no jardim. (lecture concrète et imaginative)

Maria voit les garçons jouant dans le jardin.

Maria vê sua mãe possuindo uma casa. (lecture imaginative)

Maria voit sa mère possédant une maison. 
RODRIGUES, P. A. Le gérondif comme complément des verbes...

(26) a) *Maria vê sua mãe possuir uma casa.

b) Maria vê os meninos brincar no jardim. (lecture concrète)

\section{Le temps du verbe subordonné dans un contexte irrealis}

Boivin (1998, p. 109) observe qu'en français, lorsqu'on a une interprétation imaginative du verbe perceptif, le temps de la subordonnée, bien que certaines restrictions s'imposent, peut être différent du temps de la principale, tel qu'illustré en (27):

Jean est tellement malade, je ne le vois pas aller à l'école demain.

C'est aussi le cas des compléments gérondifs en PB, c'est-à-dire que dans un contexte irrealis, le temps de la subordonnée, avec certaines restrictions, peut être différent de celui de la principale ( 28 a). Ce n'est pas le cas cependant des compléments à l'infinitif en $\mathrm{PB}$, qui ne permettent pas une lecture imaginative (28 b).

a) Maria vê os meninos brincando no jardim amanhä. Maria voit les garçons jouant dans le jardin demain.

b) *Maria vê os meninos brincar no jardim amanhā. Maria voit les garçons jouer dans le jardin demain.

\section{Conclusion}

Dans cette section, nous avons vu que les compléments gérondifs des verbes de perception en $\mathrm{PB}$ ont des caractéristiques distinctes des compléments à temps fini. Par contre, lorsqu'ils décrivent un contenu de perception concrète, les compléments au gérondif partagent plusieurs caractéristiques avec les compléments à l'infinitif et les pseudorelatives : ils impliquent la perception directe, excluent les 
RODRIGUES, P. A. Le gérondif comme complément des verbes...

prédicats statifs, et le temps de la subordonnée, ainsi que sa valeur de vérité, est dépendant de celui de la principale.

\section{L'interprétation des compléments au gérondif des verbes de perception}

Pour déterminer l'interprétation des compléments au gérondif, il est important de les comparer d'une part aux compléments infinitifs et d'autre part aux pseudorelatives, puisqu'ils partagent, comme nous venons de le voir, plusieurs caractéristiques de ces deux constructions. Nous utilisons dans notre analyse les catégories sémantiques adoptées par Labelle (1996).

Labelle montre, à partir des hypothèses de Rochette (1988) sur la correspondance syntaxe-sémantique, que les différents types de compléments phrastiques du verbe voir correspondent à des types sémantiques et syntaxiques distincts. L'auteur examine les constructions comportant des compléments à temps fini, des compléments à l'infinitif et des pseudorelatives. Les catégories sémantiques adoptées dans son travail sont les suivantes :

Action, " dont la réalisation canonique est $\mathrm{V}$, correspond à un événement réduit qui n'a pas d'existence indépendante de l'événement dénoté par le verbe principal ";

ÉVÉNEMENT, " dont la réalisation canonique est $\mathrm{I}$, correspond à une Action dont la réalisation est indépendante de l'événement décrit par le verbe principal. Il s'agit d'une description définie d'une action ou d'un état de fait qui n'a pas de valeur de vérité propre ";

Proposition, " dont la réalisation canonique est $\mathrm{C}$, correspond à un Événement dont la valeur de vérité peut être affirmée ou niée indépendamment de celle de la principale ";

Oвлет: " a comme réalisation canonique un $\mathrm{N}$ "

Pour Labelle, le complément à temps fini est sémantiquement une Proposition et syntaxiquement un $\mathrm{CP}$. Le complément à l'infinitif est sémantiquement un Événement ou une Action, parce que sa valeur de vérité n'est pas indépendante de 
celle de la principale et son temps n'est pas distinct de celui du verbe de la principale (lorsqu'il décrit un contenu de perception concrète). Quant aux pseudorelatives, elles appartiennent à la catégorie Objet et sont formées par deux constituants distincts, soit un objet suivi d'un prédicat second.

Puisque les compléments au gérondif du $\mathrm{PB}$ possèdent des caractéristiques distinctes des compléments à temps fini, nous pouvons affirmer qu'ils ne constituent pas une Proposition sémantiquement, ni un CP syntaxiquement. Dans les prochaines sections nous tentons de déterminer à quelle catégorie correspondent les compléments gérondifs : à un Objet, comme les pseudorelatives, à un Événement, comme les compléments infinitifs, ou aux deux catégories à la fois.

\section{Le complément au gérondif interprété comme un Objet}

Nous avons vu que les compléments au gérondif partagent plusieurs caractéristiques avec les pseudorelatives. Dans la section 4.1, nous tentons de déterminer si la structure syntaxique proposée par Labelle (1996) pour les pseudorelatives (le NP initial est le complément du verbe de perception et la relative forme un prédicat second) peut être appliquée aux compléments au gérondif. Ensuite, dans la section 4.2, nous discuterons d'une des structures syntaxiques proposées par Declerck (1982) pour les compléments au gérondif en anglais, soit celle où ce complément est un NP complexe.

\section{Le NP est l'objet de perception et la participiale gérondive un prédicat second}

Les verbes qui sélectionnent un NP, mais qui ne sélectionnent pas un complément phrastique, comme le verbe avistar 'apercevoir', acceptent un complément au gérondif. Les exemples en ( 29 a) et ( 29 b) nous montrent que les compléments infinitifs et le compléments à temps fini sont impossibles avec le verbe avistar; la participiale gérondive, par contre, est un complément possible (29 c). Ce fait indique que les compléments gérondifs, dans ce cas, doivent être analysés comme un objet suivi d'un prédicat second, et non comme un constituant unique dénotant un événement. 
RODRIGUES, P. A. Le gérondif comme complément des verbes...

(29) a) Nós avistamos Maria (* comprar um vestido). nous avons aperçu Maria (*acheter une robe)

b) *Nós avistamos que Maria comprava um vestido. nous avons aperçu que Maria achetait une robe

c) Nós avistamos Maria comprando um vestido. nous avons aperçu Maria achetant une robe

\section{Non-adjacence entre la participiale et son sujet de prédication}

Pour Labelle, dans le cas des pseudorelatives, si un complément locatif peut intervenir entre le NP et la relative, il est interprété comme faisant partie de la principale. Dans ce cas, on peut interpréter le NP aussi comme faisant partie de la principale :

(30) a) J'ai vu ton frère à l'atelier qui empilait des boîtes.

b) Je le voyais sur la chaussée qui perdait tout son sang.

Dans le cas des compléments au gérondif du PB, un complément locatif peut intervenir entre le NP et le gérondif; nous pouvons ainsi interpréter le NP comme faisant partie de la principale (31a). Ce n'est pas le cas des compléments infinitifs (31b) :

(31) a) Vi Maria em uma loja comprando um vestido. j'ai vu Maria dans un magasin achetant une robe.

b) *Vi Maria em uma loja comprar um vestido. j'ai vu Maria dans un magasin acheter une robe. 


\section{Coordination}

Le gérondif peut être coordonné à un prédicat second suivant un NP :

(32) Ele viu Maria preocupada e esperando por ele. il a vu Maria préoccupée et attendant lui

Les exemples que nous venons de voir nous montrent que le complément au gérondif des verbes de perception en PB peut être analysé comme étant sémantiquement un Objet, se réalisant comme un NP suivi d'un prédicat sécond.

\section{Le NP et la participiale gérondive sont dominés par un nœud NP}

Les exemples en (33) - (34) nous montrent que certains compléments au gérondif peuvent être modifiés par une relative non-restrictive.

(33) Eu vi $\int_{N P}$ a luz piscandoJ, que te incomoda tanto. $j$ 'ai vu la lumière clignotant, qui te dérange tant.

(34) Você viu $l_{N P}$ o disco contendo cançōes de Natal], que tem a capa verde?

As-tu vu le disque contenant des chansons de Noël, qui a la pochette verte?

La présence d'une relative non-restrictive dans ces exemples nous indiquent que ces compléments au gérondif doivent être analysés comme des NP complexes. L'analyse du complément gérondif comme un NP complexe ne peut toutefois pas s'appliquer à la totalité des constructions comportant ce type de complément. La phrase donnée en (35), par exemple, peut très difficilement recevoir cette analyse. 
???Vi $I_{N P}$ Maria comprando um vestido].

(je) ai vu Maria achetant une robe.

\section{Le complément au gérondif interprété comme un Événement}

Si les compléments au gérondif peuvent être considérés comme un Evénement, nous nous attendons à qu'ils se comportent comme les compléments à l'infinitif. Nous reprenons en quelques faits mentionnés par Declerck (1982) concernant la comparaison entre les compléments à l'infinitif et les compléments au gérondif en anglais; pour Declerck, le complément au gérondif est ambigu et une des ses interprétations possibles correspond à celle du complément à l'infinitif, soit un constituant unique dénotant un événement. La seule différence entre les deux concernerait l'aspect, les compléments participiaux constituant la contrepartie progressive des compléments infinitifs; le rapport existant entre ces deux constructions serait le même que celui existant entre les phrases simples et les phrases progressives. Nous reprenons des faits concernant les caractéristiques des compléments à l'infinitif mentionnés par Kleiber (1988). Nous verrons que les compléments au gérondif en PB peuvent être analysés de la même façon que les compléments à l'infinitif.

\section{Declerck (1982)}

La perception du sujet de la complétive

Selon Declerck, dans les infinitives anglaises, il n'est pas nécessaire que le sujet (NP) de la complétive soit perçu directement, parce que l'objet de perception est l'événement exprimé par le complément. Pour l'auteur, les gérondives anglaises se comportent de la même façon : nous pouvons énoncer la phrase en (36) même si John est en arrière du mur et que nous ne voyons que la scie.

(36) I saw John sawing through the wall. 
Ce fait caractérise aussi les compléments au gérondif du PB. On peut énoncer la phrase en (37) sans nécessairement voir João directement.

(37) Eu vi o João serrando os galhos da árvore.

(je) ai vu João sciant les branches de l'arbre.

\section{L'absence de sujet de perception visuelle directe}

En anglais, le complément au gérondif, ainsi que le complément à l'infinitif, est possible même en l'absence d'un sujet pouvant être perçu directement, lorsque, par exemple, le sujet est un NP abstrait (38 a). L'exemple en (38 b) nous montre que le sujet de ce type de complétive ne peut pas être passivisé, ne pouvant donc pas être considéré comme objet de perception. L'objet de perception est l'événement exprimé par la complétive.

(38) a) I have seen faith accomplish(ing) miracles.

b) *Faith has been seen (by me) accomplishing miracles.

En PB, le complément au gérondif est aussi possible dans ces cas, comme le montrent les exemples suivants:

a) ?Eu tenho visto a fé fazendo milagres. j'ai vu la foi faisant des miracles

b) *A fé tem sido vista fazendo milagres. la foi a été vue faisant des miracles

(40) a) Eu vi minhas idéias impondo-se com facilidade. j'ai vu mes idées s'imposant avec facilité

b) *Minhas idéias foram vistas impondo-se com facilidade.

mes idées ont été vues s'imposant avec facilité 


\section{Kleiber (1988)}

Kleiber (1988) compare les pseudorelatives compléments d'un verbe de perception aux compléments infinitifs. Il mentionne certaines propriétés des infinitives qui ne s'appliquent pas aux pseudorelatives, montrant que ces deux constructions ont des propriétés sémantiques différentes. Selon Labelle (1996), ces différences sémantiques sont des indices d'une différence de structure entre les infinitives, formées d'un seul constituant décrivant un Événement, et les pseudorelatives, formées de deux constituants, soit un NP suivi d'un prédicat second décrivant ce NP objet de perception au moment où il est perçu. Selon Kleiber, ces propriétés caractérisant les infinitives sont aussi propres aux compléments gérondifs. À notre avis, le fait que le complément gérondif partage les mêmes propriétés sémantiques que les compléments infinitifs est un argument en faveur de son analyse comme étant un Evénement. Dans les exemples qui suivent, les infinitives sont exemplifiées en (a), les gérondives en (b) et les pseudorelatives en (c). Les contextes présentés ci-dessous désambiguïsent les constructions gérondives, éliminant l'interprétation où la gérondive décrit un objet au moment où il est perçu et gardant l'interprétation où le complément gérondif décrit un événement.

L'infinitive et la gérondive peuvent décrire un événement prenant place dans un monde possible, tandis que l'événement perçu dénoté par la pseudorelative ne peut se passer que dans le monde réel, factuel, puisque cette construction décrit un objet de perception au moment où il est perçu:

a) Você o verá chorar.

Tu le verras pleurer.

b) Você o verá chorando.

Tu le verras pleurant.

c) *Você o verá que chorará/que chora.

*Tu le verras qui pleurera / qui pleure.

(42) a) Paul não o viu chorar.

Paul ne l'a pas vu pleurer.

b) Paul não o viu chorando.

Paul ne l'a pas vu pleurant.

c) *Paul não o viu que chorava.

*Paul ne l'a pas vu qui pleurait. 
L'infinitive et la gérondive sont compatibles avec la pluralité d'occurrences de l'événement; la pseudorelative se limite à l'expression d'une seule occurrence de perception:

a) Eu o vi várias vezes ir ao cinema. Je l'ai plusieurs fois vu aller au cinéma.

b) Eu o vi várias vezes indo ao cinema. Je l'ai plusieurs fois vu allant au cinéma.

c) *Eu o vi várias vezes que ia ao cinema. *Je l'ai plusieurs fois vu qui allait au cinéma.

L'infinitive et la gérondive sont compatibles avec la mention de la durée de l'événement et peuvent exprimer la perception d'événements achevés; la pseudorelative est nécessairement imperfective:

(44) a) Eu o vi chorar das três horas às cinco horas. Je l'ai vu pleurer de trois heures à cinq heures.

b) Eu o vi chorando das três horas às cinco horas. Je l'ai vu pleurant de trois heures à cinq heures.

c) *Eu o vi que chorava das três horas às cinco horas. *Je l'ai vu qui pleurait de trois heures à cinq heures.

L'infinitive est compatible avec la mention d'un intervalle de référence englobant la situation de perception; la pseudorelative doit être vraie à tout moment de l'intervalle de référence, ce qui entraîne la nécessité de la spécification de cet intervalle (voir l'exemple en (45 d)):

(45) a) Ontem, eu vi Paul chorar.

Hier, j'ai vu Paul pleurer.

b) Ontem, eu vi Paul chorando.

Hier, j'ai vu Paul pleurant.

c) ?Ontem, eu vi Paul que chorava.

?Hier, j'ai vu Paul qui pleurait 


\section{d) Naquele instante, eu vi Paulo que chorava. À ce moment-là, j'ai vu Paul qui pleurait.}

Les faits mentionnés dans cette section nous montrent que le complément au gérondif partage plusieurs caractéristiques avec le complément à l'infinitif. Nous suggérons que le complément au gérondif en $\mathrm{PB}$ peut par conséquent avoir la même interprétation que le complément à l'infinitif, la seule différence entre les deux étant aspectuelle, tel que l'a proposé Declerck (1981) pour l'anglais.

\section{Conclusion}

Nous avons montré dans cet article que le complément des verbes de perception construit avec un gérondif dans le PB est ambigu : il peut être interprété, d'après les catégories sémantiques proposées par Rochette (1988) et adoptées par Labelle (1996), soit comme un Événement, correspondant à un IP, soit comme un Objet, se réalisant comme un NP. Dans le cas où les compléments au gérondif sont interprétés comme $O b j e t$, ils peuvent correspondre soit à un NP suivi d'un prédicat second, soit à un NP complexe. L'analyse correspondant à un Événement semble être disponible dans la majorité des cas; on trouvera une exception dans l'exemple en (34). L'analyse où le complément gérondif correspond à un NP suivi d'un prédicat second est conditionnée au fait que la participiale gérondive doit décrire un NP objet de perception au moment où il est perçu; ainsi cette analyse n'est pas disponible pour les exemples donnés en (40-45). Il y a aussi, comme nous l'avons vu à la fin de la section 4, des restrictions quant à la possibilité d'analyser les compléments gérondifs comme un NP complexe. À notre avis, l'étude de ces différences mérite d'être approfondie.

Nous avons aussi discuté brièvement les interprétations concrète et imaginative du verbe voir. Le complément à l'infinitif en PB, qui n'a pas un sens progressif, ne permet pas une interprétation imaginative; le complément au gérondif, qui, quant à lui, a un sens clairement progressif, permet les deux interprétations. L'interprétation imaginative (irrealis ou hypothétique) semble être liée au sens progressif, tel que suggéré par Boivin (1998). Par ailleurs, elle semble également être moins restrictive par rapport au temps et à la sémantique des verbes subordonnés que l'interprétation concrète. En français, la forme infinitive permet les deux types de lecture, soit concrète et imaginative, puisque cette forme peut dénoter autant le 
sens progressif que le non progressif. Nous croyons que l'étude des aspects sémantiques concernant ces deux interprétations du verbe voir mérite d'être développée, ce qui contribuera à une meilleure compréhension de la sélection sémantique des verbes de perception.

\section{RÉSUMÉ}

Cet article a pour but de déterminer les caractéristiques sémantiques et syntaxiques des compléments des verbes de perception employés au gérondif en portugais du Brésil (PB). En utilisant plusieurs tests sémantiques et syntaxiques proposés dans la littérature, nous montrons que ce type de complément est ambigu en $\mathrm{PB}$, admettant trois analyses différentes : i) deux constituants distincts, soit un NP, l'objet du verbe de perception, suivi d'un prédicat second; ii) un constituant unique dénotant un événement; et iii) un NP complexe.

Mots-clés: Verbes de perception, complémentation, gérondif.

\section{RESUMO}

Este artigo tem como objetivo determinar as características semânticas e sintáticas dos complementos dos verbos de percepção construídos com o gerúndio em português brasileiro (PB). Utilizando diversos testes semânticos e sintáticos propostos na literatura, nós mostramos que este tipo de complemento é ambíguo em $\mathrm{PB}$, podendo receber três análises diferentes : i) dois constituintes distintos, ou seja um NP, objeto do verbo de percepção, seguido de um predicado secundário; ii) um constituinte único denotando um evento; et iii) um NP complexo.

Palavras-chave: Verbos de percepção, complementą̧ão, gerúndio.

\section{RÉFÉRENCES}

AKMAJIAN, A. The Complement structure of perception verbs in autonomous syntax framework. In: CULICOVER, P; WASOW, T.; AKMAJIAN, A. (Ed.). Formal syntax. New York: Academic Press, 1977. p. 427-460.

BOIVIN, M. C. Complementation and interpretation: the concrete and imaginative readings of 
RODRIGUES, P. A. Le gérondif comme complément des verbes...

visual perception verbs. MIT Working Papers in Linguistics, v. 25, p. 103-123, 1998.

BORGONOVO, C. Gerunds and perception verbs. Langues et Linguistique, v. 22, p. 1-19, 1996.

CARLSON, G. A unified analysis of the English bare plural. Linguistics and Philosophy, v. 1, p. 413-457, 1977.

CINQUE, G. The pseudo-relative and Acc-ing constructions after verbs of perception. In : CONFERENCE ON LOGICAL FORM OF PERCEPTION SENTENCES, 1990, Gargnano.

DECLERCK, $R$. On the role of progressive aspect in nonfinite perception verb complements, Glossa, v. 15, p. 83-114, 1981.

. The triple origin of participial perception verb complements. Linguistics Analysis, v. 10, p. 1-26, 1982 .

FELSER, C. Perception and control: a minimalist analysis of English direct perception complements. J. Linguistics, v. 34, p. 351-385, 1998.

GONÇALVES, A. Predicados complexos verbais em contextos de infinitivo não preposicionado do português europeu.1999. Thèse (Doctorat) - Université de Lisbonne.

GUASTI, M. T. Pseudorelative and prepositional infinitive. GenGenP 0-0, p. 53-65, 1992(a).

. Causative and perception verbs. Genève, 1992b. Thèse (Doctorat) - Université de Genève.

HIGGINBOTHAM, J. Les comptes rendus de perception. Communications, v. 40, p. 149-180, 1983.

KLEIBER, G. Sur les relatives du type Je le vois qui arrive. Travaux de linguistique, v. 17, p. 89$115,1988$.

LABELLE, M. Remarques sur les verbes de perception et la sous-catégorisation. Recherches Linguistiques de Vincennes, v. 25, p. 83-106, 1996.

MAURER, T. H. O infinitivo flexionado português. São Paulo: Cia. Editora Nacional, 1968.

RAPOSO, E. Prepositional infinitival constructions in European Portuguese. In: JAEGGLI, O.; SAFIR, K. (Ed.). The null subject parameter. Dordrecht: Kluwer Academic Publishers, 1989. p. 277-305.

ROCHETTE, A. Semantic and syntactic aspects of romance sentential complementation. Cambridge,1988. Thèse (Doctorat) - MIT.

SAFIR, K. Perception, selection, and structural economy. Natural Language Semantics, v. 2, p. 47-70, 1993.

VENDLER, Z. Linguistics in Philosophy. Ithaca: Cornell University Press, 1967. 